\title{
A WORKSHOP-BASED APPROACH TO TEACHING NUMERICAL METHODS AND COMPUTING IN CHEMICAL ENGINEERING
}

\author{
Jake Nease*, Kieran McKenzie, Steven Karolat, and Cynthia Pham \\ McMaster University department of Chemical Engineering \\ *Corresponding author: neasej@mcmaster.ca
}

\begin{abstract}
A workshop-based approach to teaching numerical methods in an active learning classroom is described. The strategy involves hiring student partners to design and document workshops to be used as a lecture vehicle for a class typically taught using blackboard notes. The first implementation of this method in fall 2018 yielded no significant improvements in class performance according to final grade medians and interquartile ranges, but class participation and engagement seems to have markedly improved. User satisfaction data regarding the effectiveness of the modules is generally very positive with strong support for the continued use of the modules in the future.

A cohort of students from a senior optimization class, having also used the modules in their course, shows very strong support for the further development of a workshopbased module approach to teaching numerical methods for future cohorts.
\end{abstract}

Keywords: Active learning; Class Workshops; Continuous Improvement; Student Partnerships; Numerical Methods.

\section{INTRODUCTION}

\subsection{The Motivation: Activation Energies}

Numerical methods in chemical engineering is often regarded as unapproachable, difficult, and abstract by many undergraduate engineering students at McMaster University. The root of this problem is suspected to come from the high pace of material and, owing to limited class time, a lack of in-class applications of course concepts to real-world engineering problems. These challenges, coupled with weak fundamental training in basic computing software such as Python or MATLAB prior to this course, lead to significant barriers to entry or "activation energies" for students and instructors alike. Through a combination of these barriers, student dissatisfaction and a classic (and admittedly rather ineffective) "chalk and talk" delivery style of the content, the results for this class tend to be rather poor.

A course that focuses on algorithm development, numerical complexity, and developing the right set of tools to tackle a particular problem may be better suited to a teaching style focused on discovery and applied problem solving. Through an approachable course design focusing on layered applications of concepts with embedded workshops and practice, it may be possible to catalyze the learning process, thus overcoming the activation energy of numerical methods and leading to a greater teaching and learning experience for students and instructors alike.

\subsection{The Idea: Active Learning Workshops}

The goal of this research project is to introduce active learning elements in the numerical methods classroom, including problem-based learning, collaborative activities, and in-class discussions. Extensive research has shown that active learning strategies, particularly those centered around the active-engagement of students in a traditional lecture setting, have significant impacts on student attention, performance, and satisfaction [1]. Dr. Richard Felder (a prominent name in engineering education, particularly the chemical field) among others have found that notes-with-gaps, when combined with in-class exercises and strategies to promote discussion and engagement, lead to higher student performance (grades, comprehension, and long-term retention) than students provided with complex notes or assigned textbook readings alone [2],[3],[4]. This project is aimed at developing a new lecture delivery format for a numerical methods course that leverages active learning and notes with gaps to improve student engagement and performance.

However, the strategy of including notes-with-gaps and active learning exercises in the classroom is meaningless if the focus of these activities does not meaningfully impact the learning of students. It is therefore critical to ensure that (a) the focus of these exercises results in the development of fundamental problem-solving and analysis skills rather than rote replication of calculations or repetition of concepts; and (b) the exercises are approachable in a classroom setting (meaning they can be completed in limited time with limited resources). To account for these requirements, it was decided that the individuals best suited to decide what these workshops would look like are the students themselves. 


\subsection{The Path: Students Working for Students}

One of the greatest barriers that tends to exist between instructors and students is the so-called 'curse of knowledge', which claims that the more knowledgeable someone is in a topic, the more difficult it is to explain that topic to a layman, a student, or a general audience [5]. This is not omnipresent in academia, of course, but it does provide significant motivation for including student opinions and ideas in the development of new course materials, especially for a course such as numerical methods.

To this end, the strategy for developing these workshop modules involves allowing a pair of students to develop them from the ground-up, complete with the design of inclass activities and their solutions. The instructor will then act as a reviewer, ensuring that the technical content is sound, meets the expectations of the course, and that the material can be covered in the course's allotted time.

Furthermore, it is intended that these modules and workshops therein are treated as "living" documents, where future students can continue to polish and develop the material after experiencing it first-hand in order to improve the learning experience for future cohorts.

\section{MODULE DEVELOPMENT}

The modules for this course were developed throughout the summer of 2018 and implemented for the first time in the fall of 2018. Survey data on student satisfaction combined with assessment results for the 2018 cohort versus previous cohorts was collected and will be discussed. The current versions of the modules are available via the link in the appendix.

\subsection{Brief Description of Modules}

The learning modules for numerical methods are broken into the five main thrusts of the course itself. Each section discusses any relevant theory and introduces methods for solving those types of problems. The sections are: (1) linear systems of equations; (2) nonlinear systems of equations; (3) least-squares regression and curve fitting; (4) numerical differentiation and integration; and (5) analysis and numerical integration of ordinary differential equations.

Each module is structured roughly as follows: A brief introduction to the topic provides some context for why it is important for engineering applications, typically through the descriptions of problems that employ that topic. Following this, the module enters a loop that follows the cycle idea $\rightarrow$ method $\rightarrow$ simple application $\rightarrow$ generalization $\rightarrow$ advanced application. The idea is simply the topic being introduced, of which their may be several introduced per module (i.e. linear equation systems). The method is how we are going to tackle the problem the idea presents (i.e. Gauss elimination). The simple application is typically a workshop activity that involves applying the method "by hand" to a simple problem with an emphasis on pattern recognition and generalizing the solution as much as possible (i.e. solving a small system of linear equations by hand). The generalization involves formalizing the patterns (hopefully) recognized through the initial workshop into a mathematical or algorithmic concept (i.e. describing the algorithm for forward matrix elimination). Finally, the advanced application requires the students to apply or extend the concept on their own, whether it be through a live coding workshop, or completing a derivation or an algorithm (i.e. formalizing and coding matrix back substitution).

The result of this cycle is that the students in class are a critical part of their own education and the education of others. It is typical for the advanced workshop portion of the cycle to take up to an entire class period (50 minutes), which usually means that for each major concept the students are actively applying and exploring the content roughly as much as the instructor is lecturing.

After completing the workshops, students are left with a completed set of course notes that can be used for assignments, tests, and other applications.

\subsection{Continuous Improvement}

Survey and performance data have been collected from the 2018 cohort and will be discussed in this paper. However, it is important to mention that constructive feedback from students will be used to modify the modules over the summer of 2019 by two new students (both having taken the course this past year) in order to improve the student experience going forward.

\section{METHODOLOGY}

The effectiveness of the proposed module/workshop delivery vehicle for numerical methods is assessed through a combination of quantitative class performance metrics (grades, average attendance) combined with student satisfaction and engagement results using Likert scales and written feedback.

\subsection{Quantitative Analysis}

The most accessible method of determining the impact of active learning workshops to enhance student learning of numerical methods is by comparing the results between cohorts of students before and after the change of lecture style. Data for two cohorts $(2016,2017)$ are available for the current instructor of this course prior to the workshopbased modules being implemented. One set of data (2018) is available for class performance after the workshop modules were implemented. Data available includes average class/tutorial attendance as well as performance on all assessments. Clearly, this data will continue to be collected over the coming years to further determine 
whether the new workshop-based lecture style enhances student performance.

\subsection{Qualitative Analysis}

Survey results are available for the satisfaction of the 2018 cohort regarding the use of workshop-based modules in the numerical methods classroom. Moreover, the same workshop style is used in an upper year elective class. It was thus possible to obtain the opinions of the 2017 numerical methods cohort on the potential of using workshops in the numerical methods classroom. Both data sets are presented and discussed herein. Future cohorts will continue to have data collected.

\section{RESULTS AND DISCUSSION}

The following section discusses the quantitative results of each cohort of students from 2016-2018 and the qualitative results of the 2017-2018 classes.

\subsection{Quantitative Results}

Shown below in Figure 1 are box-and-whisker plots of each final grade statistical quartile by cohort for the numerical methods class. Recall, the use of workshop modules was implemented for the 2018 cohort.

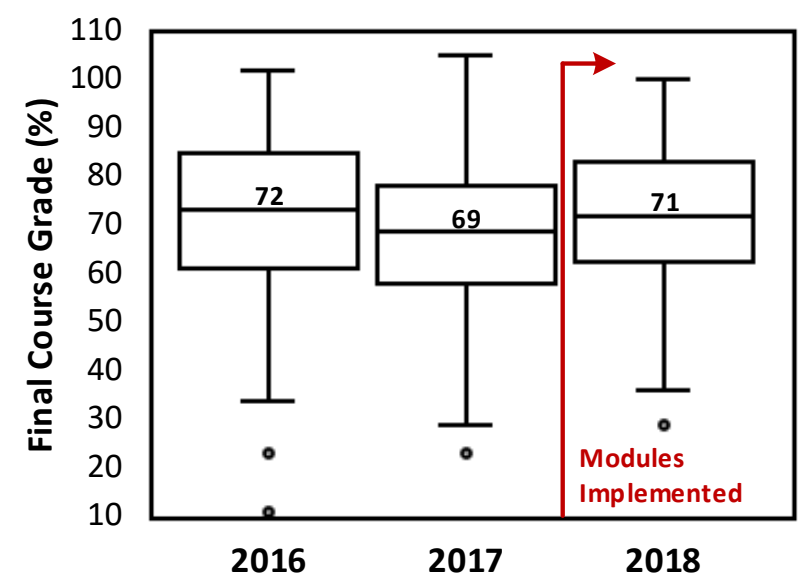

Figure 1: Final grade results for the numerical methods class for two years prior to the introduction of lecture modules and one year after. Printed scores are medians for each cohort.

It is clear from the data in Figure 1 that the overall class performance did not change significantly from 2016 to 2018, even after the introduction of the proposed workshop-based modules. Although there is not an improvement in performance, a consistent performance indicates that the workshop-based approach to lectures seems to be at least as effective as a traditional "chalk and talk" model. Since this was the first year the modules were used and significant feedback was collected in order to improve them for future offerings, there is a significant chance that future cohorts will show improved performance.
One further observation that can be made from Figure 1 is that the distribution of grades is lower when the workshop-based modules are used. This is further emphasized by a lower standard deviation in $2018(\sigma=$ $14.1 \%)$ than $2016(\sigma=15.7 \%)$. These workshops are intended to appeal to a wider range of students with various preferred learning styles and thus normalizing the performance of the "average" student by slowly bringing up the distribution of class grades while reducing variance will determine if these workshops are effective long-term.

\subsection{Contextualization of Quantitative Results}

Of course, these results must be taken with a grain of salt: It is possible for systematic and cohort bias to be affecting class performance on a year-to-year basis. For example, this course has been taught by the same instructor since 2016, meaning that the instructor's comfort with the material, expectations of students, and overall instructional ability may have improved over time. Moreover, it is very difficult to compare different cohorts of students, particularly in a competitive program where cohort attitude and performance can change form year to year. This is not meant to indicate that these results are meaningless, but rather something to keep in context as additional data is collected and more cohorts experience the workshop-based approach to lectures.

\subsection{Qualitative Results}

Perhaps just as important as the quantitative results, among the most important indicators of success for using a workshop approach to the numerical methods classroom is engagement. It is critical for the students to feel like they have had a good learning experience, which can be quantified through the collection of student satisfaction data. The following section is split into two subsections: the first describes the satisfaction of the 2018 numerical methods cohort with the modules and workshop lectures, and the second describes the opinions of the 2018/2019 classes for a fourth-year elective using these workshops on the potential of using them in the numerical methods classroom.

\section{Numerical Methods Cohort}

Shown below in Figure 2 are the opinions of students on the helpfulness of using the workshop-based modules during lecture time (white bars) and while preparing for tests and examinations (black). It can be seen in this data that the opinions of using these modules is generally quite positive, with most students indicating that they find the modules "very helpful" during class time. However, some students find the modules distracting and thus "unhelpful" during class time. Review of the written comments in the same survey indicates that some students find the modules unfocused or lengthy, which are key detractors from maintaining focus and motivation during lectures. It is also interesting to note that no students find the modules 
unhelpful when studying for tests or exams, which indicates that the students strongly value the laid-out nature of the modules and how they help assimilate and organize the course content alongside practice problems.

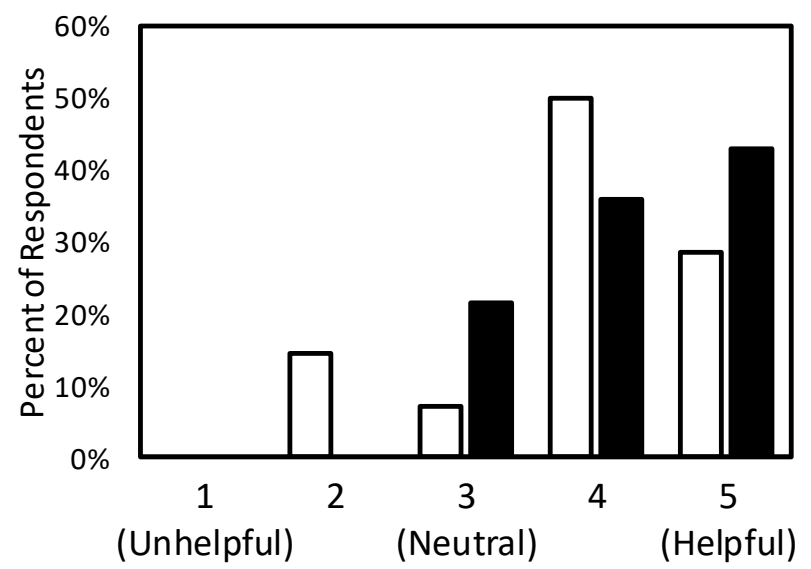

Figure 2: Survey results of the 2018 numerical methods class (15 participants) on the helpfulness of workshopbased modules during lecture (white bars) and while preparing for tests or examinations (black bars).

Shown below in Figure 3 are the comparative opinions of the 2018 numerical methods cohort on using the proposed workshop-based modules compared to two more traditional styles of instruction: slides (white) and board notes (black).

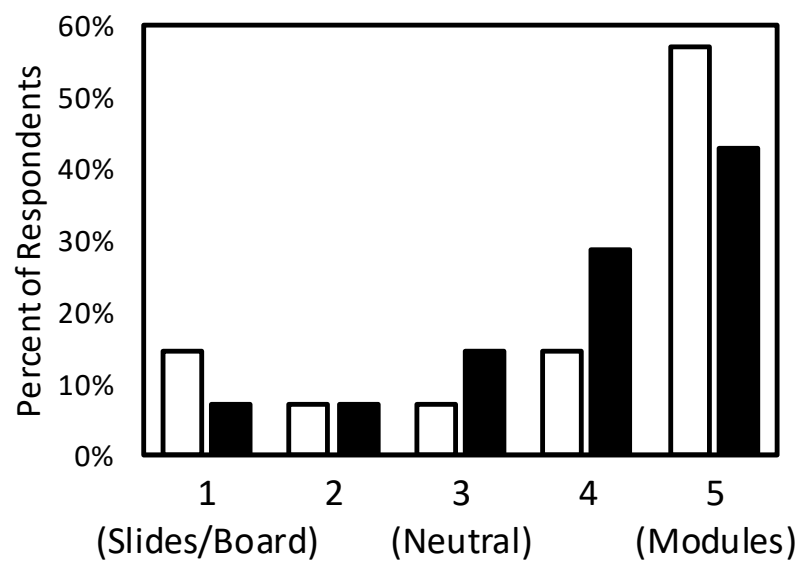

Figure 3: Survey results of the 2018 numerical methods class (15 participants) on their opinions of using workshop-based modules as a lecture vehicle versus slides (white bars) and board notes (black bars).

It is interesting to note that students have significantly varied opinions about the use of slides or chalk boards as a lecture vehicle, with some claiming that they would much rather experience those more classic forms of instruction than in-class workshops. However, it is clear once again from these results that most students prefer the workshop modules over board notes or slides, with the overwhelmingly favourite response indicating that they would strongly prefer the workshop modules if given the choice. These results indicate that the workshop approach used in the 2018 class had positive effects on student engagement.

It is also interesting to note in Figure 3 that the use of slides appears to be the most polarizing response, with several students strongly discouraging or promoting the use of slides, with very few responses the more neutral options. Overall, the use of these modules seems to appeal to most students and therefore will continue to be updated and used for future cohorts.

\section{8-2019 Optimization Cohort}

As mentioned, the fourth-year optimization course taught by the same instructor uses a similar workshopbased approach to teaching lectures. These modules are more polished than the numerical methods version thanks to a longer history and additional revisions, but the opinions of optimization students on the modules' effectiveness is nonetheless a strong way to infer their potential strengths in the numerical methods classroom. Moreover, it should be mentioned that the optimization students took the same numerical methods class using the classic "chalk and talk" delivery style (and thus can make a direct comparison).

Shown below in Figure 4 are the opinions of optimization students on the helpfulness of using the workshop-based modules during lecture time (white) and while preparing for tests and examinations (black).

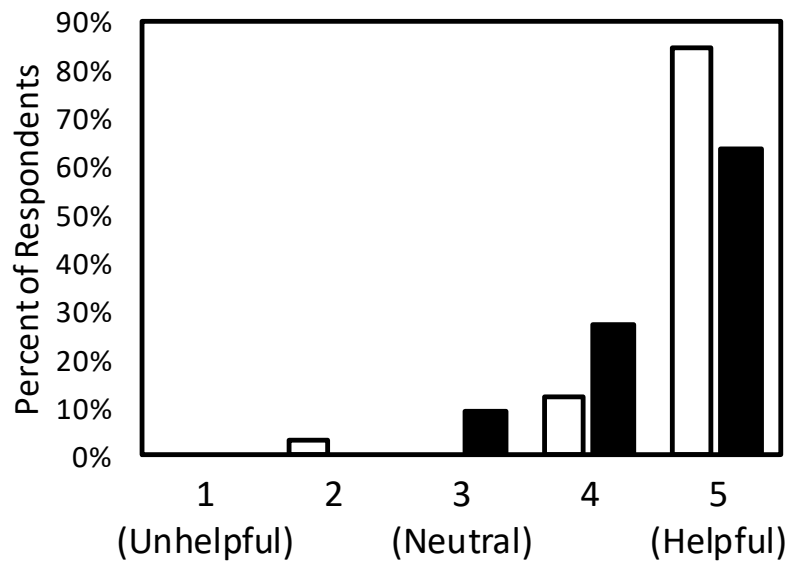

Figure 4: Survey results of the 2018-2019 optimization classes (32 participants) on the helpfulness of workshopbased modules during lecture (white bars) and while preparing for tests or examinations (black bars).

The results in Figure 4 clearly indicate the overwhelming positivity of the optimization cohort toward using the workshop modules in lecture and while preparing for tests and examinations. In fact, only one respondent indicated that the workshop modules were remotely unhelpful during lecture. Written comments indicate that the workshop-based practice problems and cycle of immediate applications after theory are the strongest assets of the workshop modules being used in the optimization classroom. Students appreciate the breaks in content, 
firming up their understanding through worked-out examples, and the ability to review previously worked out examples as additional practice for tests. It is anticipated that these modules are favoured more because they have had three rounds of student feedback and improvements versus the numerical methods versions.

Shown below in Figure 5 are the comparative opinions of the 2018-2019 optimization cohorts on using modules and workshops in lecture versus slides (white) or board notes (black).

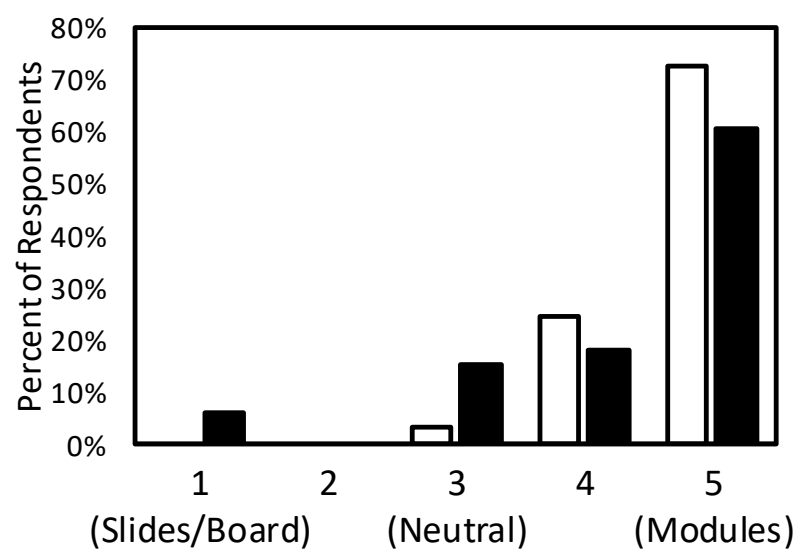

Figure 5: Survey results of the 2018-2019 optimization classes (32 participants) on their opinions of using workshop-based modules as a lecture vehicle versus slides (white bars) and board notes (black bars).

These results clearly show a preference for using workshop modules in class with over $79 \%$ of responses landing in the 4+ category for both slides and board notes. Written comments from students indicate that the modules provide a "best of both worlds" scenario in which dry content is already laid out for digestion (such as on slides) but students must also stay attentive to complete in class examples (as they would have to if these examples were performed on the board). It is interesting to note several responses indicating that they would strongly prefer board notes over the modules; this is likely a subset of students that enjoyed the numerical methods class the first time around and clearly do not think it should be changed. Otherwise, students seem to feel that the use of modulebased workshops in the optimization classroom is favourable to more traditional lecture formats.

One final piece of data that may be collected from the optimization cohort is whether they, based on prior experience, would have preferred their numerical methods class to be taught using workshop modules. Having experienced two very similar courses each using different styles, this can provide motivation for the continued refinement of the modules for the numerical methods class. Shown in Figure 6 are the opinions of the optimization class on using workshop modules in the numerical methods class based on their undergraduate experiences.

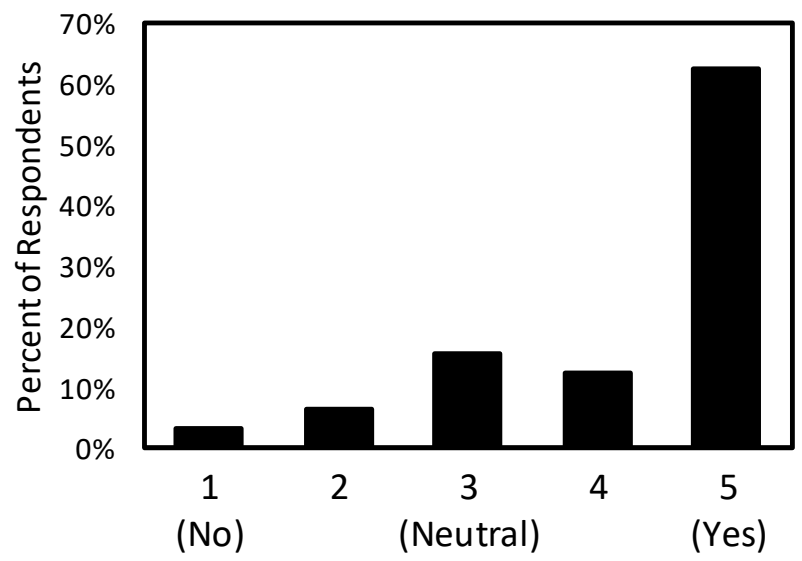

Figure 6: Opinions of the 2018-2019 optimization cohorts on the use of modules in numerical methods.

It can be seen in the figure above that $63 \%$ of students strongly support the use of workshop modules for numerical methods, and $76 \%$ support them overall. $16 \%$ of students are neutral on the concepts and less than $10 \%$ would recommend against it. To have over three quarters of the class indicate that their learning experience would have been improved by using workshop modules in their numerical methods class is strong evidence that the modules are an effective lecture and study tool and should be continued to be developed in the future.

\section{CONCLUSIONS AND FUTURE WORK}

Along with an excellent group of undergraduate students, a workshop-based approach to learning numerical methods was constructed in the summer of 2018 and delivered in the fall of 2018 with overall success. Class performance on a quantitative basis was not significantly affected by the shift in classroom methods, although the consistent performance was considered acceptable when combined with improved attendance (not tracked in 2018), student engagement and overall satisfaction.

Data regarding student satisfaction with the modules was collected with a variety of Likert scales from the 2018 numerical methods class and the 2018-2019 cohorts of a fourth-year optimization course using the same workshopbased approach to learning. In general, the modules were favourably reviewed by all cohorts ( $\geq 50 \%$ favourable reviews on all fronts) with positive opinions on the usefulness of the modules for lecture engagement and for evaluation preparation. Moreover, senior students strongly recommended the use of workshop modules in the numerical methods classroom to enhance student learning and satisfaction.

Future work will be, first and foremost, to refine and improve upon the existing numerical methods modules by incorporating student feedback and organizing focus groups to brainstorm ways to improve the content and delivery of the workshop modules. Additionally, the 
refined modules will be used for the 2019 numerical methods cohort with additional metrics such as class attendance and performance on conceptual versus numerical evaluations will be recorded and analyzed. It is the final goal of this project to produce open-educational resources (OERs) out of these modules so that they may be used universally to teach any second- or third-year university course on numerical computing for engineering.

\section{Acknowledgements}

Funding for the project was gratefully provided by the McMaster MacPherson Leadership in Teaching and Learning (LTL) Grant, the McMaster department of Chemical Engineering, and the Associate Dean's Research office of the McMaster faculty of Engineering.

\section{References}

[1] Prince, M. (2004). Does Active Learning Work? A Review of the Research. Journal of Engineering Education, 93: 223-231. doi:10.1002/j.21689830.2004.tb00809.x.

[2] Felder, R.M., Brent, R. (2016). Teaching and Learning in STEM: A Practical Guide. John Wiley \& Sons. ISBN $1118925823,9781118925829$.
[3] Cornelius, T.L. Owen-DeSchryver, J. (2008). Differential Effects of Full and Partial Notes on Learning Outcomes and Attendance, Teaching of Psychology, 35:1, 6-12, DOI: 10.1080/00986280701818466.

[4] Richard M. Felder and Linda K. Silverman, "Learning and teaching styles in engineering education," Engineering Education, vol. 78, no. 7, pp. 674-681, 1988. Available as of March 3, 2012, from http://winbev.pbworks.com/f/LS-1988.pdf

[5] Heath, C., \& Heath, D. (2007). Made To Stick. The Random House Publishing Group.

\section{APPENDIX A: LIVING DOCUMENTS}

The numerical methods workshops and corresponding modules are intended to be provided as open educational resources once completed. For the time being, interested parties may access the most up-to-date versions of the modules via the following link: https://mcmasteru365my.sharepoint.com/:f:/g/personal/neasej_mcmaster_ca/Eq vBXTwGpDVAmcMHsaVVfscBaVuCJimdIzVsQUsrG8 nV2A? $=$ QuSgMo 